\title{
Experiments on the Speed of Light
}

\author{
José R. Croca' ${ }^{1,2}$, Rui Moreira', Mário Gatta1,3, Paulo Castro ${ }^{1}$ \\ ${ }^{1}$ Center for Philosophy of Sciences of the University of Lisbon (CFCUL), Lisbon, Portugal \\ ${ }^{2}$ Department of Physics, Faculty of Sciences, University of Lisbon, Lisbon, Portugal \\ ${ }^{3} \mathrm{CINAV}$ and Escola Naval (Portuguese Naval Academy), Lisbon, Portugal \\ Email: jpcastro@fc.ul.pt
}

How to cite this paper: Croca, J.R., Moreira, R., Gatta, M. and Castro, P. (2019) Experiments on the Speed of Light. Journal of Applied Mathematics and Physics, 7, 1240-1249.

https://doi.org/10.4236/jamp.2019.75084

Received: February 21, 2019

Accepted: May 28, 2019

Published: May 31, 2019

Copyright (c) 2019 by author(s) and Scientific Research Publishing Inc. This work is licensed under the Creative Commons Attribution International License (CC BY 4.0).

http://creativecommons.org/licenses/by/4.0/

\begin{abstract}
Many experiments concerning the determination of the speed of light have been proposed and done. Here two important experiments, Michelson-Morley and Sagnac, will be discussed. A linear moving variation of Michelson-Morley and Sagnac devices will then be proposed for probing experimentally the invariance of the speed of light.
\end{abstract}

\section{Keywords}

Speed of Light, Michelson-Morley Experiment, Sagnac Experiment, One-Arm Linear Moving Michelson-Morley and One-Beam Sagnac Interferometer Variants

\section{Introduction}

Starting with Galileo, many experiments to study the speed of light have been proposed and done. One of the most famous, designed to study the existence of a hypothetical luminiferous aether, was undoubtedly the Michelson and Morley experiment [1]. The null results of this experiment, to prove the existence of a hypothetical aether wind, led to relativity [2].

Another very important set of experiments, although less acknowledged by most of the scientific community, was done by Sagnac [3] [4] [5] in 1910. The results of his experiments are, according to some authors, against the assumption of the impossibility of attaining speeds for the light greater than $c$, in every physical circumstance.

A natural conclusion of the Sagnac experiment and of other modern improvements of it done by Ruyong and collaborators [6] [7] is that indeed it seems possible, at least according to some authors, to go beyond the limit imposed by relativity for signal transmission. On the other hand, further increasing this belief, the tunneling experiments by Nimtz [8] [9] [10], reproduced over the 
world, have also led to information transmission at velocities up to $v>4.7 c$. This experimental evidence seems to indicate that in very special cases it is indeed possible to attain speeds beyond the light barrier.

A linear one-arm moving version of Michelson-Morley experiment that shares, in a certain way, very close similarities with a one-beam linear variant of Sagnac experiment will be presented here.

\section{The Michelson-Morley Experiment}

The Michelson-Morley device is one of the long list of experimental setups designed to study the behavior of the speed of the light in diverse physical situations. The sketch of the experimental setup is shown in Figure 1 (whenever possible, we have drawn the light rays paths separately in the depicted experiments).

The drawing represents the best possible situation for a possible alteration of the interference pattern observed at the detector, in which the device is standing still relative to the Earth and the horizontal arm of the interferometer is aligned with the hypothetical luminous aether wind. For other less affected directions, measurements were made at well separated times, taking in consideration the rotation of the Earth relative to the aether or, simply by rotating the interferometer.

In the best situation, shown in the picture above, for the hypothetical effect of the luminiferous aether wind to manifest itself on the velocity of light, the expected experimental results under the hypotheses stated below, would be:

1) The luminous aether exists. Assume further that the effect of the aether wind on the speed of light is described by a linear additive rule, $v=c \pm v_{A e}$. Here $v$ is the resultant light velocity, $c=3 \times 10^{8} \mathrm{~m} / \mathrm{s}$ is the speed of the light in the air or in vacuum, and $v_{A e}$ is the velocity of the aether relative to the Earth.

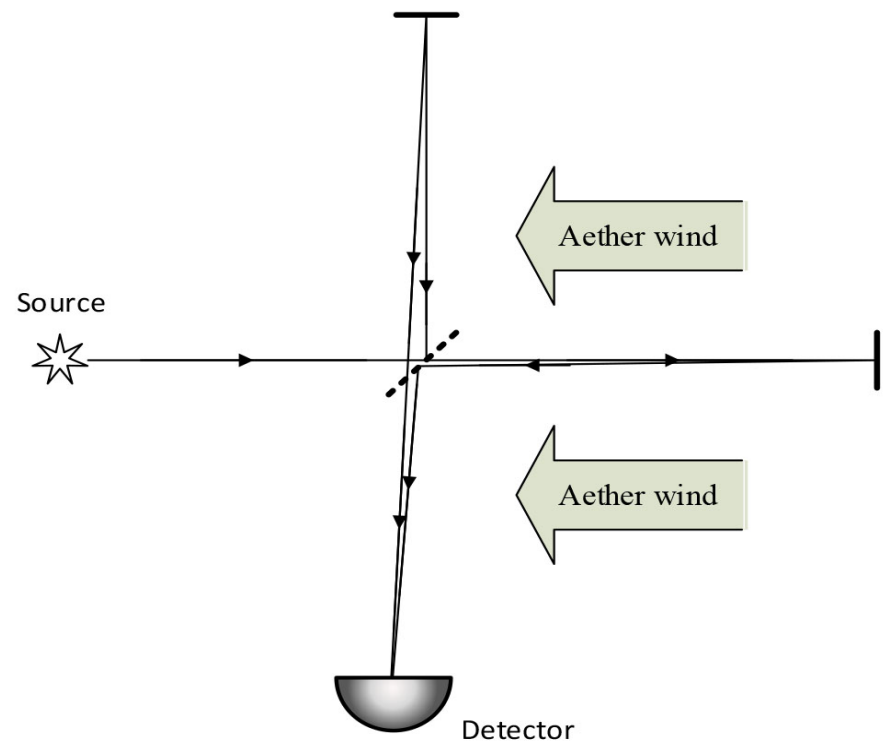

Figure 1. The Michelson-Morley experiment. 
In these circumstances the expected times taken in each direction would be:

$$
t_{1}=\frac{L}{c-v_{A e}} \text { and } t_{2}=\frac{L}{c+v_{A e}}
$$

$L$ being the length of both the horizontal and the vertical interferometer arms.

The total time taken, for the light to go and return along the horizontal arm, beamsplitter-mirror-beamsplitter, is given by

$$
t_{h}=t_{1}+t_{2}=\frac{L}{c-v_{A e}}+\frac{L}{c+v_{A e}}
$$

The reference time, taken as the round time from the splitting region along the vertical arm, will be the same in each direction and given approximately by

$$
t_{v}=t_{1}^{\prime}+t_{2}^{\prime}=\frac{L}{c}+\frac{L}{c}=2 \frac{L}{c}
$$

The time difference seen at the detector is

$$
\Delta t=\frac{L}{c-v_{A e}}+\frac{L}{c+v_{A e}}-2 \frac{L}{c}
$$

This was the approach followed by Michelson and Morley. Other authors have improved on their calculations along the vertical arm leading to the final result

$$
\Delta t=\frac{L}{c-v_{A e}}+\frac{L}{c+v_{A e}}-2 \frac{L}{\sqrt{c^{2}-v_{A e}^{2}}}
$$

The time difference between the two beams arriving at the detector would lead to an observable change in the interference pattern, both with (4) and (5).

2) No luminous aether exists or if it exists does not affect significantly the speed of light.

In this situation $v_{A e}=0$ and consequently the time difference between the round trip of light in both arms of the interferometer will be $\Delta t=0$.

As is well known, experiments done by Michelson and Morley and later improved upon by many experimental teams, all over the world, have shown no significant change in the fringe pattern, implying that indeed we have $\Delta t=0$. This means that no aether exists or if it does, it has no observable effect on the behavior of light. As it is well known, these "null" results led Einstein to his theory of relativity.

\section{The Sagnac Experiment}

Instead of leaving the interferometer standing still with the Earth, and thus moving relatively to a hypothetical luminiferous aether in which it is immersed, Sagnac placed his device on a rotating platform, as shown in Figure 2

In order to estimate the expected time difference between the two round tips, again the two previous assumptions are possible:

1) The linear additive rule applies. In this situation, the times taken by each round trip, depending on the respective propagation direction relative to the rotation of the apparatus, would be given by 


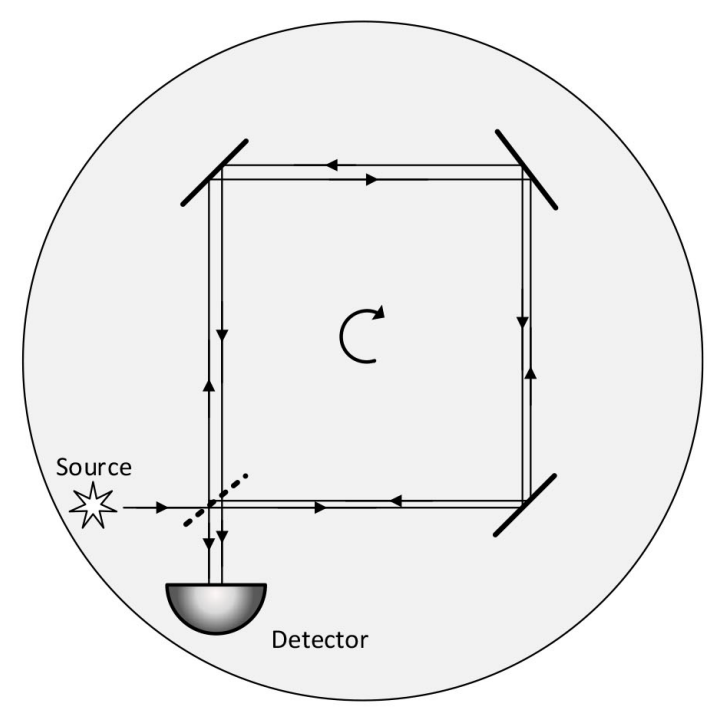

Figure 2. The Sagnac experiment.

$$
t_{1}=4 \frac{\frac{L}{4}}{c-v_{\ell}}=\frac{L}{c-v_{\ell}} \text { and } t_{2}=4 \frac{\frac{L}{4}}{c+v_{\ell}}=\frac{L}{c+v_{\ell}}
$$

and the time difference

$$
\Delta t=t_{1}-t_{2}=\frac{L}{c-v_{\ell}}-\frac{L}{c+v_{\ell}}=L\left(\frac{1}{c-v_{\ell}}-\frac{1}{c+v_{\ell}}\right)
$$

in which $v_{\ell}$ is the tangential velocity of the mirrors and of the beamsplitter, and $L$ is the total length of the light along the square path.

2) The linear additive rule does not apply and no change in the speed of the light is expected in any direction. In this case, we have $t_{1}=t_{2}$ and consequently we shall have a null time difference, that is

$$
\Delta t=t_{1}-t_{2}=0
$$

Experiments confirm that the additive linear rule applies and that the observed time difference is consequently given by expression (7).

These surprising experimental result have been explained both by relativists [11] [12], that assume that the speed of light can never, in any conceivable circumstance, be greater than $c,(v \leq c)$, and by the authors [13] [14] who believe that in Nature there are no "man-made impossibilities". Consequently, one thing that seems truly impossible, at a certain time and in a given theoretical and experimental context, in another more developed framework may turn out to be perfectly possible. In such circumstances, it would be possible to have indeed superluminal velocities, such as $v=c+v_{\ell}>c$.

To determine if the observed Sagnac time deviation was due to the rotation of the device, thus originating some acceleration effect, Ruyong and his collaborators [6] [7] developed what they called a linear variant of the Sagnac interferometer, in which the enclosed area may eventually be null. Their idea was, instead of a rotating platform, to use an optical fiber moving along a loop in which 
source and detector would move together with the fiber, as shown in the picture below, Figure 3. Initially, they used the traditional circular optical ring obtaining, naturally, the same results as Sagnac did. Next, they placed the moving optical fiber loop between two wheels with the same angular velocity. They made devices with different sizes, up to fifty meters.

The results of these experiments, done for different linear sizes of the device, always with the same angular velocity of the wheels, show that there exists indeed a direct dependence of the observed time difference with the length of the apparatus, given by expression (7). Consequently, one concludes that it is not the rotation (here solely at the ends of the conveyor) that is essential to the observed light velocities, but rather the linear parts of the motion of the optical fibers. There is some resemblance here to the twin paradox [15], in many instances wrongly attributed to the accelerations of the travelling twin (see also, e.g., Adler, Bazin and Schiffer [16], as well as Maudlin [17]).

\section{The Modified Michelson-Morley Experiment}

Inspired in the ideas of Sagnac and Ryuong, it is possible to conceive an interesting modification of the Michelson-Morley experiment. The idea is very simple! Place the Michelson-Morley interferometer-source mirrors and detector-on a linearly moving platform. The platform could be placed in diverse moving systems such as: an airplane, a train, or other, as shown in Figure 4.

Let us now see what are the predictions for the experiment.

Assuming that:

1.1) The speed of the light does not depend on the motion of the source relative to any referential, namely the Earth.

1.2) The linear motion is relative. In this case one may consider the platform supporting the device as standing still and the exterior medium moving or vice-versa.

1.3) Between inertial frames of reference it is not possible to distinguish which is in motion since their relative velocity is constant, $v_{i}=$ Constant.

1.4) In such conditions, we may take the platform and the interferometer as standing still and the exterior medium moving.
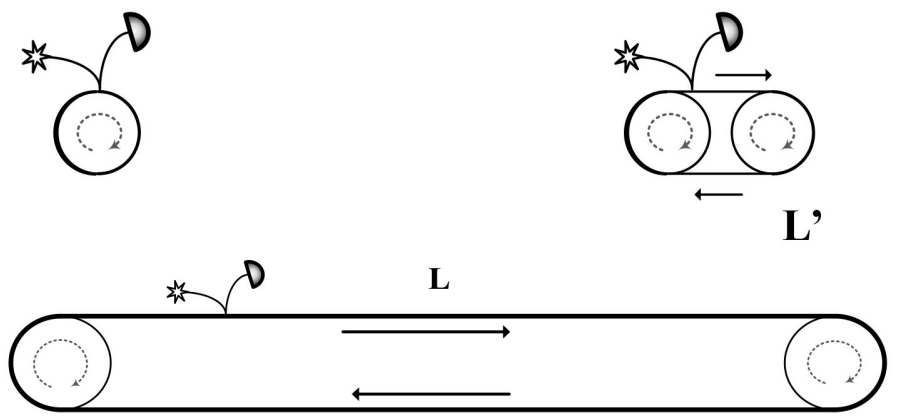

Figure 3. Linear Sagnac interferometer with different sizes (the star represents the laser source and the $\mathrm{D}$-shaped symbol represents the detector). 


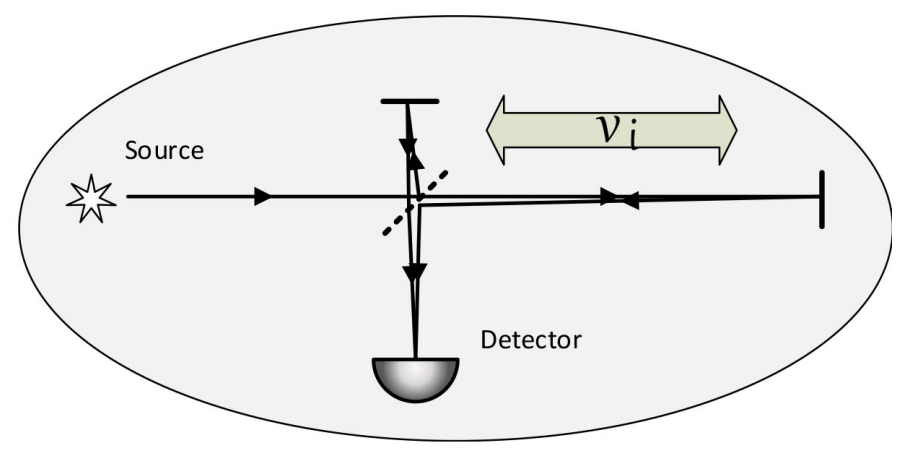

Figure 4. Moving asymmetric Michelson-Morley interferometer.

As a result of these assumptions, no difference in the observed transit times is to be expected, and $\Delta t=0$.

2) The light, that is, the physical being called photon is a complex quantum entity.

Thus, in certain particular interacting situations with the medium, we may observe speeds greater than $v>c$ and the Galilean linear additive rule will describe fairly well what is going on.

Accordingly, along the line of motion of the platform, the expected observed times taken in each direction would then be:

$$
t_{1}=\frac{L}{c-v_{i}} \text { and } t_{2}=\frac{L}{c+v_{i}}
$$

with $L$ being the length of the longitudinal interferometer arm.

The total time taken for the light to go and return along the horizontal arm, $t_{h}$, beamsplitter-mirror-beamsplitter, is now given by

$$
t_{h}=t_{1}+t_{2}=\frac{L}{c-v_{i}}+\frac{L}{c+v_{i}}=2 \frac{L}{c} \frac{1}{1-v_{i}^{2} / c^{2}}
$$

The reference time $t_{v}$, which is the round time along the vertical arm, whose size is

$$
L^{\prime}=\alpha L \quad \text { with } 0 \leq \alpha \leq 1
$$

may be approached by

$$
t_{v}=t_{1}^{\prime}+t_{2}^{\prime}=\frac{\alpha L}{c}+\frac{\alpha L}{c}=2 \frac{\alpha L}{c}
$$

Because the asymmetric arm may be very small, eventually reaching zero when $\alpha=0$, the time difference seen at the detector will be

$$
\Delta t=t_{h}-t_{v}=\frac{L}{c-v_{i}}+\frac{L}{c+v_{i}}-2 \frac{\alpha L}{c}
$$

which, for $\alpha=0 \rightarrow t_{v} \cong 0$, gives

$$
\Delta t=\frac{L}{c-v_{i}}+\frac{L}{c+v_{i}}
$$

At this point it is convenient to add that, in order to be possible to observe in- 
terferences, it is necessary for the coherence length of the source $\sigma$ to be greater than the round trip length of the light along the horizontal arm of the interferometer, that is:

$$
\sigma \geq 2 L
$$

\section{Modified Interferometers}

The Michelson-Morley and Sagnac interferometers essentially compare the time difference of two beams of light approximately traveling along the same optical distance. At the time of their development this requirement was very imperative since the coherence length of the available thermal light sources was very small. Now, due the development of high stable laser sources, with large coherence length, this requirement is no longer needed. Naturally, in any circumstance the optical path difference may exceed the coherence length of the source.

This new experimental setups makes it possible have one-arm Michelson-Morley and one-beam Sagnac interferometers.

\subsection{One-Arm Michelson-Morley Interferometer}

The asymmetric Michelson-Morley interferometer transforms into a one-arm interferometer when the vertical path becomes zero, see Figure 5.

As stated above, the coherence length $\sigma$ of the light source verifies condition (15).

Furthermore, assuming that there is a significant interaction of the photonic quantum of light with the moving medium, adequately described by an additive rule, the expected time difference is given by the expression

$$
\Delta t=t_{h}-t_{v}=t_{1}+t_{2}-0=\frac{L}{c-v_{i}}+\frac{L}{c+v_{i}}
$$

in which $t_{h}=t_{1}+t_{2}$ and $t_{v}=0$.

On the contrary, if the velocity of the light is indeed, in every conceivable circumstance, independent on the velocity of the interferometer, we will have

$$
\Delta t \neq \Delta t\left(v_{i}\right)
$$

\subsection{One-Beam Sagnac Interferometer}

The one-beam Sagnac interferometer, as the name suggests, has one single beam of light traveling in the rotating platform, as shown in Figure 6.

For this experimental setup, the expected time difference between the round-trip beam and the (almost) direct beam is simply given by

$$
\Delta t=\frac{L}{c+v_{\ell}} \text { or else } \Delta t=\frac{L}{c-v_{\ell}}
$$

at the detector, depending on the relative direction of the motion between the platform and the light beam ( $L$ being, as before, the perimeter of the squared light path). 


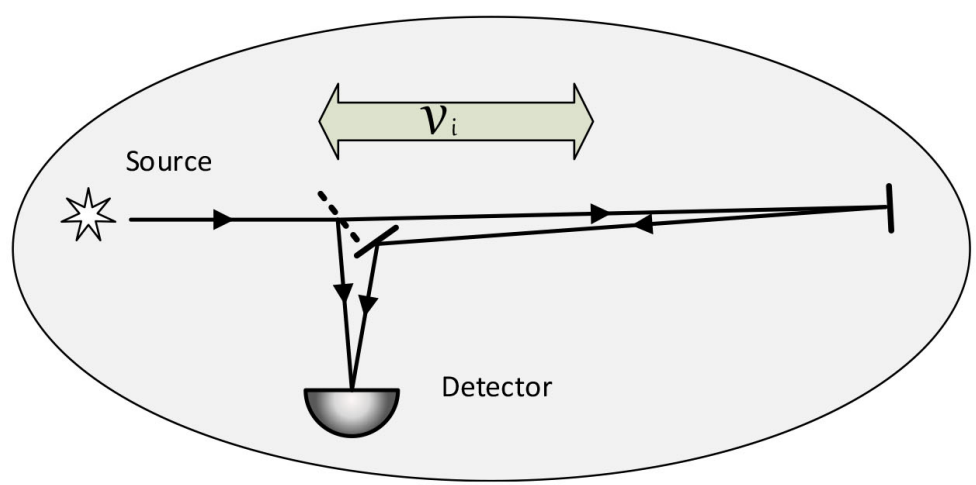

Figure 5. One arm Michelson-Morley interferometer.

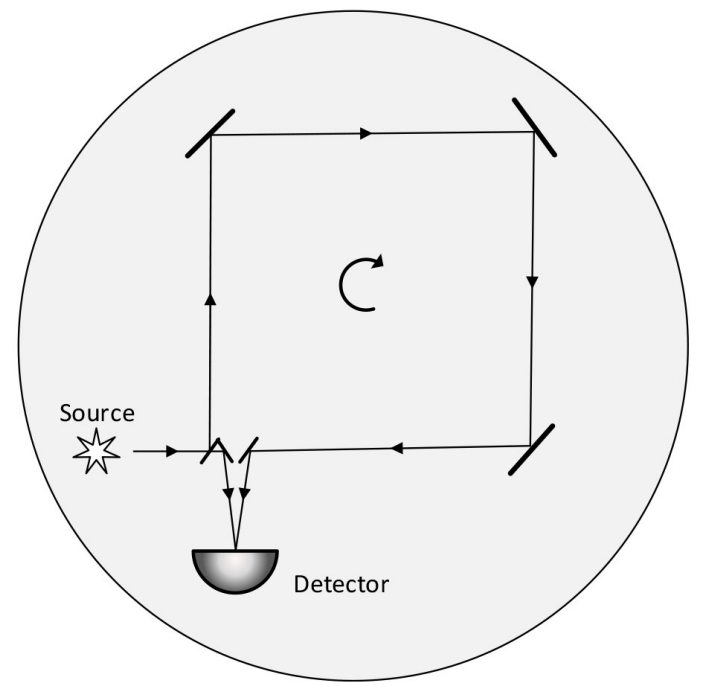

Figure 6. One-beam Sagnac interferometer.

This device could be stretched and placed on a linear moving platform (but now without rotation), as in Figure 7, next.

Again, assuming that the linear additive rule describes approximately the experimental situation, the expected time difference, will be given by

$$
\Delta t=\frac{L / 2}{c+v_{i}}+\frac{L / 2}{c-v_{i}}
$$

This one-beam moving Sagnac interferometer is, essentially, a one-arm Michelson interferometer, as depicted in Figure 5.

On the other hand, it should be noted that this device is, in a certain sense, very similar to the ingenious device imagined by Ryoung, Figure 3. Only now we do not have optical fibers in rotation and it is rather the whole device that is moving along a rectilinear trajectory.

\section{Conclusion}

The proposed modified interferometers, in principle, should allow for a relatively easy detection of any possible change in the speed of light, depending on the 


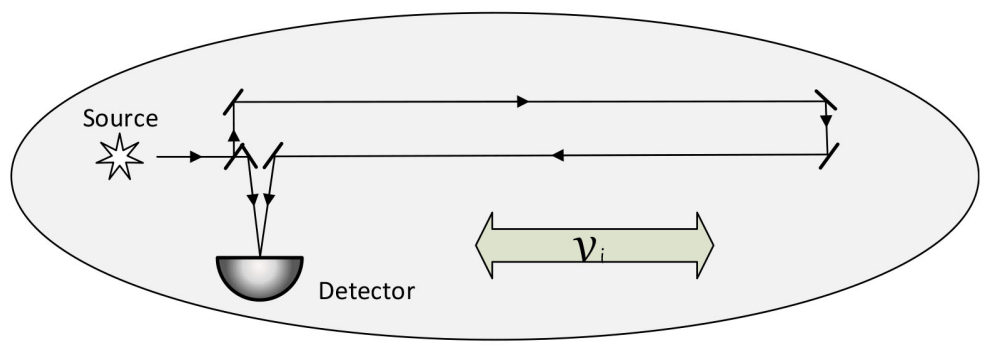

Figure 7. One-beam Sagnac interferometer on a linearly moving platform (each horizontal path has now a length $L / 2$ ).

rectilinear speed of the interferometer relative to the Earth, and without taking into consideration any hypothetical acceleration effects.

\section{Acknowledgements}

This work has been funded by FCT (Portugal) through CFCUL (project UID/FIL/00678/2019).

\section{Conflicts of Interest}

The authors declare no conflicts of interest regarding the publication of this paper.

\section{References}

[1] Michelson, A.A. and Morley, E.W. (1887) On the Relative Motion of the Earth and the Luminiferous Ether. American Journal of Science, 34, 333-345.

https://doi.org/10.2475/ajs.s3-34.203.333

[2] Einstein, A. (1905) On the Electrodynamics of Moving Bodies, Translation from Zur Elektrodynamik bewegter Körper. Annalen der Physik, 322, 891-921. https://doi.org/10.1002/andp.19053221004

[3] Sagnac, G.M.M. (1913) L'éther lumineux démontré par l'effet du vent relatif d'éther dans un interféromètre en rotation uniforme. Comptes Rendus de 1 Académie des Sciences, 157, 708-710.

[4] Sagnac, G.M.M. (1913) Sur la preuve de la réalité de l'éther lumineux par l'expérience de l'interférographe tournant. Comptes Rendus de PAcadémie des Sciences, 157, 1410-1413.

[5] Sagnac, G.M.M. (1914) Effet tourbillonnaire optique. La circulation de l'éther lumineux dans un interférographe tournant. Journal of Physics. Theories and Applications, 4, 177-195.

[6] Wang, R., Zheng, Y., Yao, A. and Langley, D. (2003) Modified Sagnac Experiment for Measuring Travel-Time Difference between Counter-Propagating Light Beams in a Uniformly Moving Fiber. Physics Letters A, 312, 7-10. https://doi.org/10.1016/S0375-9601(03)00575-9

[7] Wang, R., Zheng, Y. and Yao, A. (2004) Generalized Sagnac Effect. Physical Review Letters, 93, Article ID: 143901. https://doi.org/10.1103/PhysRevLett.93.143901

[8] Nimtz, G., Enders, A. and Spieker, H. (1993) Photonic Tunneling Experiments: Superluminal Tunneling. In: Van der Merwe, A. and Garuccio, A., Eds., Wave and Particle in Light and Matter, Plenum, New York. 
[9] Nimtz, G. and Stahlhofen, A.A. (2007) Macroscopic Violation of Special Relativity. arXiv: 0708.0681v1 [quant-ph].

[10] Nimtz, G. and Bruney, P. (2018) On the Universal Scattering Time of Neutrons. arXiv: 1807.06934 [physics gen-phy].

[11] Langevin, P. (1921) Compt. Rend, 173, 831.

[12] Langevin, P. (1937) Compt. Rend, 205, 304.

[13] Croca, J.R. (2015) Eurhythmic Physics, or Hyperphysics. The Unification of Physics. Lambert, Berlin.

[14] Croca, J.R. (2003) Towards a Nonlinear Quantum Physics. World Scientific, London. https://doi.org/10.1142/9789812776334

[15] Selleri, F. (2005) Absolute Velocity Resolution of the Clock Paradox. In: Dvoeglazov, V., Ed., Einstein and Poincaré. The Physical Vacuum, Apeiron, Montreal, 35-44.

[16] Adler, R., Bazin, M. and Schiffer, M. (1975) Introduction to General Relativity. 2nd Edition, McGraw-Hill I.S.E., Tokyo.

[17] Maudlin, T. (2012) Philosophy of Physics-Space and Time. Princeton University Press, Princeton and Oxford. 